\title{
COVID-19 vaccine safety monitoring in Republic of Korea from February 26, 2021 to October 31, 2021
}

\author{
Insob Hwang ${ }^{\circledR}$, Kyeongeun Park ${ }^{(}$, Tae Eun Kim ${ }^{\circledR}$, Yunhyung Kwon ${ }^{\circledR}$, Yeon-Kyeng Lee ${ }^{(1)}$ \\ Adverse Event Management Team, Post-vaccination Management Group, COVID-19 Vaccination Task Force, Korea Disease Control and \\ Prevention Agency, Cheongju, Korea
}

Received: November 15, 2021

Revised: December 6, 2021

Accepted: December 8, 2021

Corresponding author:

Yeon-Kyeng Lee

Adverse Event Management

Team, Post-vaccination Management Group, COVID-19

Vaccination Task Force,

Korea Disease Control and

Prevention Agency, 187

Osongsaengmyeong 2-ro,

Osong-eup, Heungdeok-gu,

Cheongju 28159, Korea

E-mail: yeonkyenglee@cdc.go.kr

\section{ABSTRACT}

Objectives: This study aimed to present data on reported adverse events following coronavirus disease 2019 (COVID-19) vaccination in Republic of Korea from February 26 to October 31, 2021, and to determine whether any significant patterns emerged from an analysis of the characteristics of suspected adverse event cases for each type of vaccine.

Methods: Adverse events following COVID-19 vaccination reported by medical doctors and forensic pathologists were analyzed. Cases of suspected anaphylaxis were classified using the Brighton Collaboration definition.

Results: By October 31, 2021, a total of 353,535 (0.45\%) adverse events were reported after 78,416,802 COVID-19 vaccine doses. Of the adverse events, $96.4 \%$ were non-serious and $3.6 \%$ were serious. The most frequently reported adverse events were headache, myalgia, and dizziness. Of the 835 reported deaths after COVID-19 vaccination, 2 vaccine-related deaths were confirmed. Suspected anaphylaxis was confirmed in 454 cases using the Brighton Collaboration definition.

Conclusion: The commonly reported symptoms were similar to those described in clinical trials. Most reported adverse events were non-serious, and the reporting rate of adverse events following COVID-19 vaccination was higher in women than in men (581 vs. 315 per 100,000 vaccinations). Confirmed anaphylaxis was reported in 5.8 cases per $1,000,000$ vaccinations.

Keywords: COVID-19; Safety; Vaccines

\section{Introduction}

The first vaccines against coronavirus disease 2019 (COVID-19) introduced in Korea were the AstraZeneca COVID-19 vaccine (ChAdOx1 nCoV-19), a viral vector vaccine, and the PfizerBioNTech COVID-19 vaccine (BNT162b2), an mRNA vaccine. These vaccines were approved for use as 2 doses for those aged $\geq 18$ years by the Ministry of Food and Drug Safety on February 10, 2021 and March 5, 2021, respectively, and were recommended by the Korea Advisory 
Committee on Immunization Practices (KACIP). Next, the Janssen COVID-19 vaccine (Ad26.COV2.S) and the Moderna COVID-19 vaccine (CX-024414) were approved on April 7 and May 21, 2021, respectively. Thus, 4 COVID-19 vaccines are currently being used in the vaccination campaign in Korea. In accordance with the vaccination plan announced on January 28, 2021, vaccination was started on February 26; however, following European reports of an association between the AstraZeneca COVID-19 vaccine and rare thrombosis with thrombocytopenia syndrome (TTS), the recommended age group eligible for this vaccine was changed to $>30$ years on April 12, 2021 and then to $>50$ years on June 29, 2021 [1-3]. At the time of establishing the vaccination plan, adolescents aged $\leq 17$ years were excluded. On August 25, the KACIP recommended vaccination for children aged 12 to 17 years and pregnant women. In Korea, in accordance with the Infectious Disease Control and Prevention Act, doctors and forensic pathologists report adverse events following immunization (AEFI) to the webbased COVID-19 vaccination management system regardless of the clinical severity of the event or the determination of causality. Reported adverse events are classified as serious if they involve hospitalization in the intensive care unit, life-threatening illness, permanent disability, a congenital anomaly, a birth defect, or death.

This study collected and analyzed data on adverse events after COVID-19 vaccination to provide an early warning that the safety of COVID-19 vaccines may require further investigation and to detect any possible new side effects.

\section{Materials and Methods}

From February 26, 2021, when COVID-19 vaccination began, to October 31, 2021, 78,416,802 doses were administered in Korea. The characteristics of the 353,535 adverse events reported on the COVID-19 vaccination management system were analyzed by sex, age, and vaccine type. Suspected cases of anaphylaxis were classified into 5 levels using the World Health Organization Brighton Collaboration definition, and cases of levels 1 through 3 were classified as anaphylaxis [4]. Since the current activity was conducted and authorized by the Korea Disease Control and Prevention Agency, the purpose was to disseminate information to the public, and the data are presented in aggregated format, the current study falls under a category of exemptions from the government regulations for ethical board review.

\section{Results}

From February 26, 2021 to October 31, 2021, 353,535 adverse events were reported, with a reporting rate of $0.45 \%$. Of these, 340,715 (96.4\%) were common adverse events such as myalgia, fever, and headache, and 12,820 cases (3.6\%) were serious adverse events such as admission to the intensive care unit or death.

The reporting rate of adverse events per 100,000 vaccinations was 581 for women and 315 for men, corresponding to a rate approximately half of that of women. The reporting rate by age group was highest in the age group of 20 to 29 years (515 per 100,000 doses), and the reporting rate decreased as age increased. The reporting rate among those aged $>75$ years was 280 per 100,000 doses. The adverse event reporting rate by vaccine type was 522 per 100,000 doses after the AstraZeneca COVID-19 vaccine, 368 per 100,000 doses after the Pfizer-BioNTech COVID-19 vaccine, 623 per 100,000 doses after the Moderna COVID-19 vaccine, and 575 per 100,000 doses after the Janssen vaccine (Table 1).

The AstraZeneca COVID-19 vaccine showed the largest difference in the reporting rate according to age and the dose administered. In particular, the reporting rate of adverse events was very high after the first vaccination in those aged $<30$ years, whereas it was lower among those aged $\geq 30$ years, and those aged $\geq 75$ years had a relatively low reporting rate compared to the other 2 COVID-19 vaccines (Figure 1 ). The current status of vaccination by age is presented in the supplementary (Table S1). By October 31, 21,994,707 first doses of the Pfizer-BioNTech COVID-19 vaccine and 22,501,177 second doses were administered. The Pfizer-BioNTech COVID-19 vaccine showed a different pattern from the AstraZenica COVID-19 vaccine in terms of adverse events by age and dose. A high rate of reported adverse events was only observed after the second dose in those aged $\geq 75$ years. Moreover, the adverse event reporting rate by age group was relatively consistent, unlike what was observed for AstraZeneca (Figure 1).

Although the most frequent symptoms were slightly different for each vaccine, the top 3 most frequently reported adverse events were headache, myalgia, and dizziness (Figure 2). The distribution of adverse event symptoms can be found in the supplementary (Table S2).

Of the 1,400 suspected cases of anaphylaxis that were reported, 1,278 cases were evaluated by provincial rapid response teams. Of these, 454 cases were confirmed as anaphylaxis through a case evaluation according to the anaphylaxis pictorial level of certainty algorithm and review by the vaccination damage investigation team, and approximately 5.8 cases per 1,000,000 vaccinations (454 cases out of 78,416,802 doses) were reported. By vaccine type, 4.2 cases per 1,000,000 doses occurred after the AstraZeneca COVID-19 vaccine, 6.6 cases per 1,000,000 doses after the 
Table 1. Reports of adverse events after receipt of COVID-19 vaccines, by recipients' sex, age group, and type of vaccine: Republic of Korea, February 26, 2021 to October 31, $2021(n=353,535)$

\begin{tabular}{|c|c|c|c|c|c|c|}
\hline \multirow{3}{*}{ Variable } & \multirow{3}{*}{$\begin{array}{c}\text { Vaccination } \\
\text { dose }\end{array}$} & \multicolumn{5}{|c|}{ Adverse events reported $^{a)}($ per 100,000$)$} \\
\hline & & \multirow{2}{*}{ Sub-total } & \multirow{2}{*}{$\begin{array}{l}\text { Non-serious } \\
\text { adverse event }\end{array}$} & \multicolumn{3}{|c|}{ Serious adverse event ${ }^{c}$} \\
\hline & & & & Death & Anaphylaxis & Others \\
\hline Total & $78,416,802$ & $353,535(451)$ & $340,715(434)$ & $835(1)$ & $1,400(2)$ & $10,585(13)$ \\
\hline \multicolumn{7}{|l|}{ Sex } \\
\hline Female & $40,018,486$ & $232,683(581)$ & $225,463(563)$ & $319(1)$ & $959(2)$ & $5,942(15)$ \\
\hline Male & $38,398,316$ & $120,852(315)$ & $115,252(300)$ & $516(1)$ & $441(1)$ & $4,643(12)$ \\
\hline \multicolumn{7}{|l|}{ Age group (y) } \\
\hline$\leq 19$ & $2,207,056$ & $8,998(408)$ & $8,788(398)$ & $1(<1)$ & $54(2)$ & $155(7)$ \\
\hline $20-29$ & $11,503,735$ & $59,283(515)$ & $58,018(504)$ & $18(<1)$ & $366(3)$ & $881(8)$ \\
\hline $30-49$ & $24,527,651$ & $117,615(480)$ & $114,426(467)$ & $73(<1)$ & $605(2)$ & $2,511(10)$ \\
\hline $50-74$ & $33,388,114$ & $148,608(445)$ & $142,361(426)$ & $377(1)$ & $318(1)$ & $5,552(17)$ \\
\hline$\geq 75$ & $6,790,246$ & $19,031(280)$ & $17,122(252)$ & $366(5)$ & $57(1)$ & $1,486(22)$ \\
\hline AstraZeneca vaccine & $20,342,226$ & $106,287(522)$ & $101,028(497)$ & $348(2)$ & $313(2)$ & $4,598(23)$ \\
\hline 1st dose & $11,109,331$ & $82,428(742)$ & $78,454(706)$ & $223(1)$ & $275(1)$ & $3,476(17)$ \\
\hline 2nd dose & $9,232,895$ & $23,859(258)$ & $22,574(244)$ & $125(1)$ & $38(0)$ & $1,122(12)$ \\
\hline Pfizer-BioNTech & $44,577,886$ & $163,920(368)$ & $157,892(354)$ & $427(1)$ & $828(2)$ & $4,773(11)$ \\
\hline 1st dose & $21,994,707$ & $89,615(407)$ & $85,915(391)$ & $228(1)$ & $660(3)$ & $2,812(13)$ \\
\hline 2nd dose & $22,501,177$ & $74,114(329)$ & 71,788 (319) & $199(1)$ & $168(1)$ & $1,959(9)$ \\
\hline 3rd dose & 82,002 & $191(233)$ & $189(230)$ & 0 & 0 & $2(2)$ \\
\hline Moderna & $12,010,627$ & $74,788(623)$ & $73,621(613)$ & $48(<1)$ & $211(2)$ & 908 (8) \\
\hline 1st dose & $6,556,837$ & $39,418(601)$ & $38,550(588)$ & $30(<1)$ & $180(3)$ & $658(10)$ \\
\hline 2nd dose & $5,453,736$ & $35,370(649)$ & $35,071(643)$ & $18(<1)$ & $31(1)$ & $250(5)$ \\
\hline 3rd dose & 54 & 0 & 0 & 0 & 0 & 0 \\
\hline Janssen & $1,486,063$ & $8,540(575)$ & $8,174(550)$ & $12(1)$ & $48(3)$ & $306(21)$ \\
\hline
\end{tabular}

COVID-19, coronavirus disease 2019.

a) Data were calculated using information on suspected adverse events after COVID-19 vaccination reported by medical facilities or doctors. The results do not suggest causality between the vaccines and adverse events. ${ }^{b}$ Non-serious adverse events include the following: common symptoms such as redness at the site of injection, pain, swelling, muscle pain, fever, headache, chills, and others. ${ }^{c)}$ Serious adverse events include the following: death, suspected anaphylaxis, and major adverse events (adverse events of special interest, admission in the intensive care unit, life-threatening events, permanent disability/sequelae, and others).

Pfizer-BioNTech COVID-19 vaccine, 14.1 cases per 1,000,000 doses after the Janssen vaccine, and 4.3 cases per 1,000,000 doses after the Moderna COVID-19 vaccine. The median time of anaphylaxis symptom onset was 10 minutes after vaccination, and $83.9 \%$ of cases occurred within 30 minutes of vaccination (Tables 2 and 3 ).

The number of deaths reported after vaccination was 835. The number of reported deaths was slightly higher in men than in women, and deaths were mostly reported in older age groups. In rapid responses to cases of death, the Vaccine Injury Investigation Committee (VIIC) evaluated the causality between vaccination and death based on medical records, death certificates, autopsy results, and epidemiological investigation results. To date, among the death cases, there have been 2 cases of confirmed causality: 1 case of myocarditis after the Pfizer-BioNTech COVID-19 vaccine and 1 case of TTS after the AstraZeneca COVID-19 vaccine.

\section{Discussion}

In Korea, 2 types of COVID-19 vaccines (AstraZeneca and Pfizer-BioNTech) were initially administered in priority groups such as residents of long-term care hospitals and facilities, health care workers, and the elderly. After that, Janssen and Moderna COVID-19 vaccines were also rolled out. The type of vaccine was allocated to specific groups in accordance with the recommended conditions of use as follows: the Pfizer-BioNTech COVID-19 vaccine for those 75 years and older with mobility, who were required to visit vaccination centers, and the AstraZeneca vaccine for residents of long-term care facilities who received on-site vaccination. The KACIP then changed the age recommendation for the COVID-19 vaccines due to a possible link between the 
Insob Hwang et al.
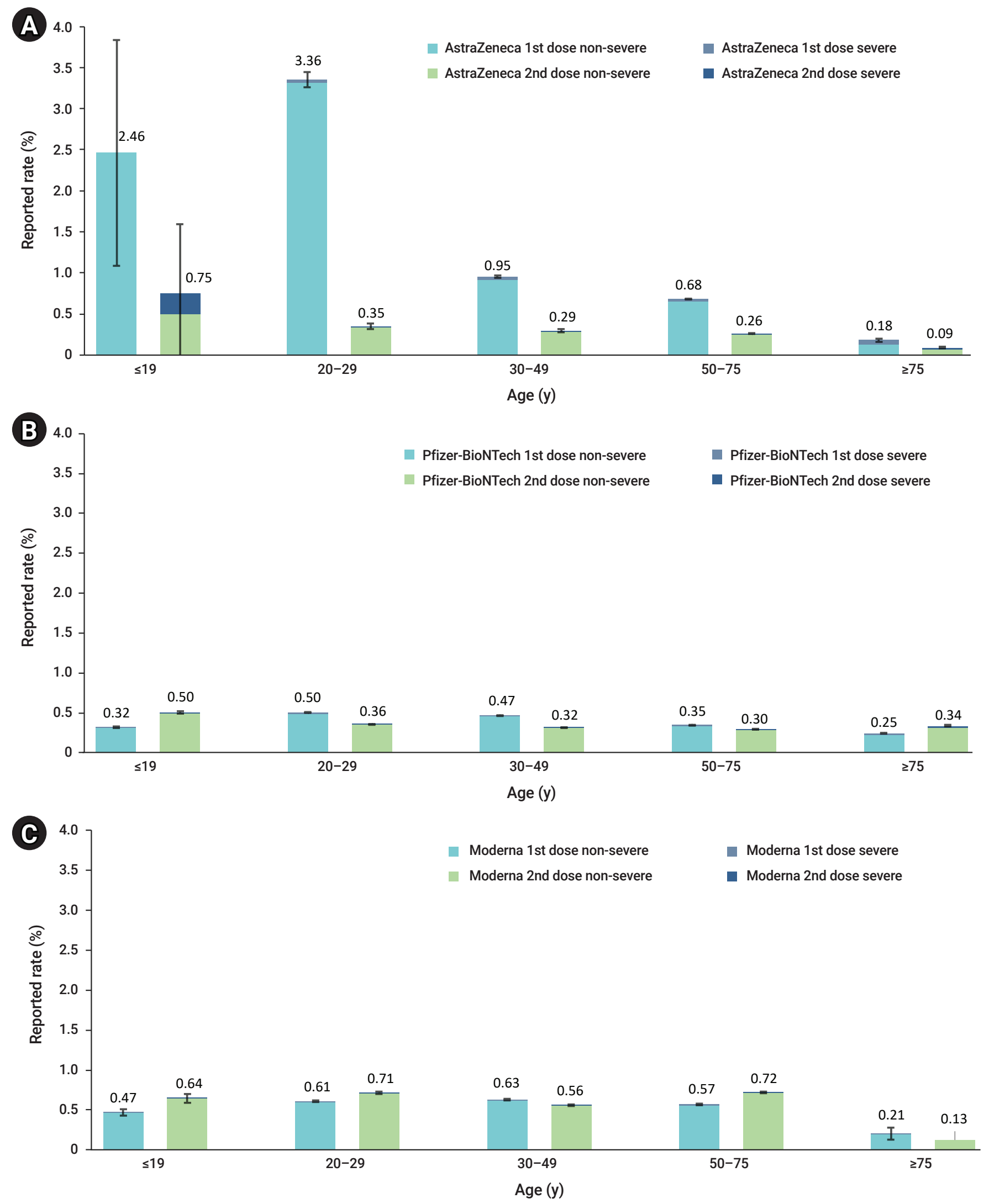

Figure 1. Adverse events reporting rates following coronavirus disease 2019 (COVID-19) vaccination by age group (February 26 to October 31, 2021).

Error bars represent 95\% confidence intervals. (A) AstraZeneca, (B) Pfizer-BioNTech, (C) Moderna. 

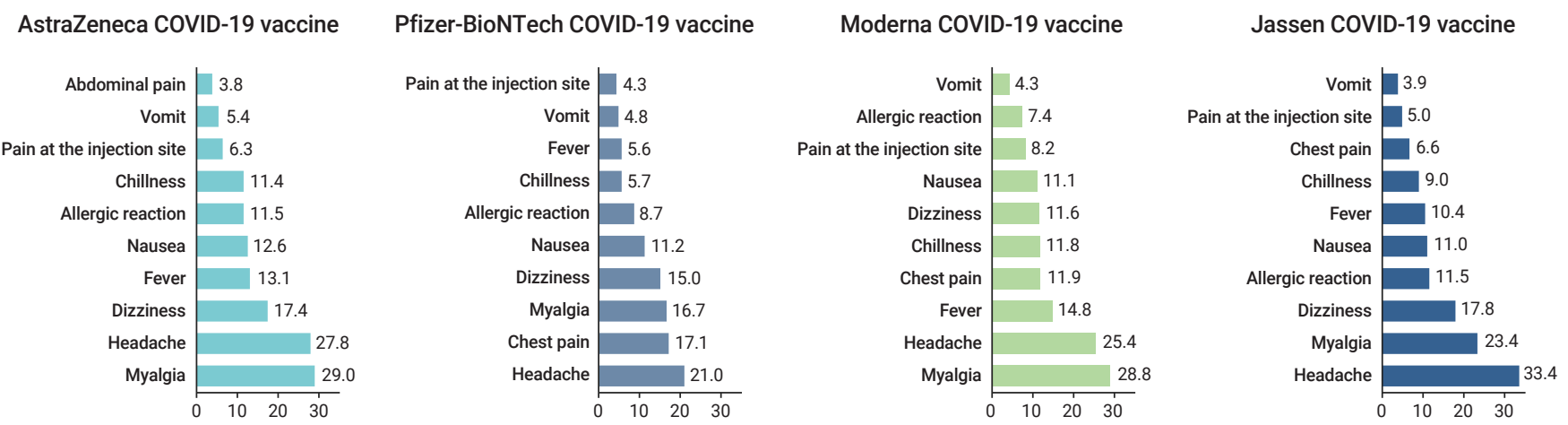

Figure 2. The proportion of symptoms of adverse events following coronavirus disease 2019 (COVID-19) vaccination by vaccine type (February 26 to October 31, 2021).

Values represent percentages and include duplicated reports.

Table 2. Classification of anaphylaxis and anaphylaxis-like acute allergic reactions in recipients of COVID-19 vaccines using the Brighton Collaboration case definition, Republic of Korea, February 26 to October 31, 2021, Republic of Korea Brighton Collaboration case definition level

\begin{tabular}{lccccc}
\hline Vaccine doses & $\begin{array}{c}\text { Total } \\
(n=78,416,802)\end{array}$ & $\begin{array}{c}\text { AstraZeneca } \\
(n=20,342,226)\end{array}$ & $\begin{array}{c}\text { Pfizer-BioNTech } \\
(n=44,577,886)\end{array}$ & $\begin{array}{c}\text { Moderna } \\
(n=12,010,627)\end{array}$ & $\begin{array}{c}\text { Janssen } \\
(n=1,486,063)\end{array}$ \\
\hline Total & 1,278 & 296 & 790 & 144 & 48 \\
Levels 1-3 (per 1,000,000) & $454(5.8)$ & $85(4.2)$ & $296(6.6)$ & $52(4.3)$ & $21(14.1)$ \\
Level 4 & 627 & 131 & 403 & 72 & 21 \\
Level 5 & 197 & 80 & 91 & 20 & 6 \\
\hline
\end{tabular}

The Brighton Collaboration case definition uses combinations of symptoms to define levels of diagnostic certainty. Levels 1 to 3 represent the highest level of diagnostic certainty that a reported case represents anaphylaxis (with level $1>$ level $2>$ level 3 ); level 4 is a case reported as anaphylaxis with insufficient evidence to meet any of the levels of diagnostic certainty; and level 5 is a case that did not meet the case definition (not a case of anaphylaxis) [1]. This study considered levels 1,2 , and 3 as anaphylaxis cases.

COVID-19, coronavirus disease 2019.

COVID-19 vaccine and adverse events.

As of October 31, 2021, the rate of adverse events was $0.45 \%$ out of $78,416,802$ doses administered. As has been described in the results from clinical trials and reports from other countries, vaccine recipients can expect non-serious adverse events during the early post-vaccination stage, including myalgia, headache, fever, and pain at the site of injection [5,6]. In addition, the reporting rate decreased from $1.8 \%$ in the first week of vaccination to $0.45 \%$ in the 35th week [7].

Reviewing the 835 deaths reported after COVID-19 vaccination after October 31, 2021, it was found that most of the individuals who died were older adults or had underlying medical conditions; their deaths were not related to the COVID-19 vaccine. Similar to a previous report from the United States [8], anaphylaxis had a higher incidence among women, with 8.3 cases per million doses (320 out of $38,398,316$ ) compared to 3.3 cases per million doses (134 out of $40,018,486$ ) in men. Furthermore, $68.1 \%$ of confirmed anaphylaxis cases occurred within 15 minutes; therefore, the previous recommendation that all recipients should be monitored for 15 to 30 minutes after vaccination in an observation room at vaccination sites should be adjusted.

This study has some limitations. The data for adverse events reported by medical institutions might underestimate the actual incidence because they are reported only for those who visited medical institutions. However, AEFIs for COVID-19 vaccinations are relatively likely to be reported since they relate to newly introduced vaccines. Compared to previous reports of adverse events after mass vaccinations during the 2009 H1N1 influenza epidemic in Korea, far more cases are being reported now. Not all reported symptoms and diagnoses were verified or confirmed by the VIIC. The AEFI cases reported in Korea are similar to those reported in the United Kingdom [5].

According to the World Health Organization, the widespread use of COVID-19 vaccines, including in the elderly and in patients with underlying health conditions, will unfortunately mean that there will be deaths and cases of serious illness that are purely coincidental and unrelated to vaccinations [9]. Since the beginning of COVID-19 vaccination, more than 3.2 billion people have already been vaccinated worldwide. 
Table 3. Characteristics of confirmed cases of anaphylaxis following COVID-19 vaccination, Republic of Korea, February 26 to October 31,2021

\begin{tabular}{|c|c|c|c|c|c|}
\hline Characteristic & $\begin{array}{l}\text { AstraZeneca } \\
\quad(n=85)\end{array}$ & $\begin{array}{l}\text { Pfizer-BioNTech } \\
\quad(n=296)\end{array}$ & $\begin{array}{l}\text { Janssen } \\
(n=21)\end{array}$ & $\begin{array}{l}\text { Moderna } \\
(n=52)\end{array}$ & $\begin{array}{c}\text { Total } \\
(n=454)\end{array}$ \\
\hline Age group (y) & $51(21-87)$ & $34.5(17-91)$ & $37(30-51)$ & $30.5(19-51)$ & $37(17-91)$ \\
\hline$\leq 19$ & NR & $18(6.1)$ & NR & $2(3.9)$ & $20(4.4)$ \\
\hline $20-29$ & $12(14.1)$ & $100(33.8)$ & NR & $21(40.4)$ & $133(29.3)$ \\
\hline $30-49$ & $27(31.8)$ & $120(40.5)$ & $19(90.5)$ & $28(53.8)$ & $194(42.7)$ \\
\hline $50-74$ & $45(52.9)$ & $36(12.2)$ & $2(9.5)$ & $1(1.9)$ & $84(18.5)$ \\
\hline$\geq 75$ & $1(1.2)$ & $22(7.4)$ & NR & NR & $23(5.1)$ \\
\hline \multicolumn{6}{|l|}{ Sex } \\
\hline Female & $71(83.5)$ & $210(70.9)$ & $6(28.6)$ & $33(63.5)$ & $320(70.5)$ \\
\hline Male & $14(16.5)$ & $86(29.1)$ & $15(71.4)$ & 19 (36.5) & $134(29.5)$ \\
\hline Symptom onset ${ }^{a)}$ & $10(1-1,200)$ & $10(0-900)$ & $5(1-530)$ & $10.5(1-210)$ & $10(0-1,200)$ \\
\hline$\leq 15 \mathrm{~min}$ & $58(68.2)$ & $199(67.2)$ & $18(85.7)$ & $34(65.4)$ & $309(68.1)$ \\
\hline$\leq 30 \mathrm{~min}$ & $66(77.6)$ & $248(83.8)$ & $20(95.2)$ & $47(90.4)$ & 381 (83.9) \\
\hline$>30 \mathrm{~min}$ & $19(22.4)$ & $48(16.2)$ & $1(4.8)$ & $5(9.6)$ & $73(16.1)$ \\
\hline \multicolumn{6}{|l|}{ Prior allergic reaction } \\
\hline Yes & $35(41.2)$ & $91(30.7)$ & $3(14.3)$ & $15(28.8)$ & $144(31.7)$ \\
\hline No & $50(58.8)$ & 205 (69.3) & $18(85.7)$ & $37(71.2)$ & $310(68.3)$ \\
\hline \multicolumn{6}{|l|}{ Symptom } \\
\hline Sensation of throat closure & $35(41.2)$ & $114(38.5)$ & $7(33.3)$ & $20(38.5)$ & $176(38.8)$ \\
\hline Upper airway swelling & $16(18.8)$ & $34(11.5)$ & $1(4.8)$ & $3(5.8)$ & $54(11.9)$ \\
\hline Nausea/vomiting & $40(47.1)$ & $114(38.5)$ & $13(61.9)$ & $18(34.6)$ & $185(40.7)$ \\
\hline Tachycardia & $32(37.6)$ & $79(26.7)$ & $5(23.8)$ & $17(32.7)$ & $133(29.3)$ \\
\hline $\begin{array}{l}\text { Difficulty breathing without } \\
\text { wheeze or stridor }\end{array}$ & $34(40.0)$ & $58(19.6)$ & $1(4.8)$ & $12(23.1)$ & $105(23.1)$ \\
\hline Angio-edema & $20(23.5)$ & $46(15.5)$ & $3(14.2)$ & $5(9.6)$ & $74(16.3)$ \\
\hline Hypotension & $29(34.1)$ & $133(44.9)$ & $12(57.1)$ & $28(53.8)$ & $202(44.5)$ \\
\hline Decreased level of consciousness & $13(15.3)$ & 40 (13.5) & $4(19.0)$ & $11(21.2)$ & $68(15.0)$ \\
\hline Other & $4(4.7)$ & $35(11.8)$ & $2(9.5)$ & $1(1.9)$ & $42(9.3)$ \\
\hline \multicolumn{6}{|l|}{ Treatments received } \\
\hline Epinephrine & $60(70.5)$ & $209(70.6)$ & $19(90.5)$ & $29(55.8$ & $317(69.8)$ \\
\hline Anti-histamine & $54(63.5)$ & $143(48.3)$ & $12(57.1)$ & $26(50.0)$ & $235(51.8)$ \\
\hline Steroid & $46(54.1)$ & $132(44.6)$ & $9(42.8)$ & $24(46.2)$ & $211(46.5)$ \\
\hline Other & $5(5.9)$ & $92(31.1)$ & $1(4.8)$ & $6(11.5)$ & $104(22.9)$ \\
\hline \multicolumn{6}{|l|}{ Treatment progress } \\
\hline Hospitalized & $13(15.3)$ & $32(10.8)$ & NR & $5(9.6)$ & $50(11.0)$ \\
\hline Outpatient/emergency room & $72(84.7)$ & $264(89.2)$ & $21(100)$ & $47(90.4)$ & $404(89.0)$ \\
\hline
\end{tabular}

Data are presented as median (range) or $n(\%)$.

COVID-19, coronavirus disease 2019; NR, not reported.

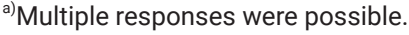

It has been established that the AstraZeneca and Janssen COVID-19 vaccines are associated with TTS, a very rare condition [10]. Considering that COVID-19 vaccines are new, it is necessary to regularly scrutinize the causal relationship between reported adverse events and COVID-19 vaccines and whether the number of observed AEFIs exceeds that of expected cases by chance.

\section{Supplementary Material}

Table S1. COVID-19 vaccines administered by age group; Table S2. Symptoms after the first and second doses of COVID-19 vaccines by vaccine type (per 100,000 doses). Supplementary data are available at https://doi.org/10.24171/ j.phrp.2021.0310. 


\section{Notes}

\section{Ethics Approval}

Since the current activity was conducted and authorized by the public health authorities, and the purpose was to disseminate information to the public, the current study was exempted from ethical board review.

\section{Conflicts of Interest}

The authors have no conflicts of interest to declare.

\section{Funding}

None.

\section{Availability of Data}

The data used in this study is protected under the Personal Information Protection Act.

\section{Authors' Contributions}

Conceptualization: YK, YKL; Data curation: IH, KP; Formal analysis: IH, TEK; Investigation: IH, TEK; Methodology: KP, YK; Validation: YK, YKL; Writing-original draft: IH, YK; Writing-review $\&$ editing: all authors.

\section{Additional Contributions}

We thank the relevant ministries (including the Ministry of Interior and Safety), cities and provinces, medical staff in health centers, and medical facilities for their efforts in responding to vaccine safety monitoring.

\section{References}

1. Korea Disease Control and Prevention Agency (KDCA). Update on COVID-19 and COVID-19 vaccination. Cheongju: KDCA; 2021.

2. European Medicines Agency (EMA). Press briefing on the conclusion of the assessment of the Pharmacovigilance Risk Assessment Committee (PRAC) of Vaxzevria (previously COVID-19 Vaccine AstraZeneca) and thromboembolic events. Amsterdam: EMA; 2021.
3. Korea Disease Control and Prevention Agency (KDCA). Updated action plan for COVID-19 vaccination 2021. Cheongju: KDCA; 2021.

4. Lee E, Lee YK, Kim TE, et al. Reports of anaphylaxis after coronavirus disease 2019 vaccination, South Korea, 26 February to 30 April 2021. Euro Surveill 2021;26:2100694.

5. Medicines and Healthcare Products Regulatory Agency (MHRA) Summary of yellow card reporting [Internet]. MHRA; 2021 [cited 2021 Nov 15]. Available from: https://www.gov.uk/government/ publications/coronavirus-covid-19-vaccine-adverse-reactions/ coronavirus-vaccine-summary-of-yellow-card-reporting.

6. Gee J, Marquez P, Su J, et al. First month of COVID-19 vaccine safety monitoring-United States, December 14, 2020-January 13, 2021. MMWR Morb Mortal Wkly Rep 2021;70:283-8.

7. Oh HK, Kim EK, Hwang I, et al. COVID-19 vaccine safety monitoring in the Republic of Korea: February 26, 2021 to April 30, 2021. Osong Public Health Res Perspect 2021;12:264-8.

8. Shimabukuro TT, Cole M, Su JR. Reports of Anaphylaxis After Receipt of mRNA COVID-19 Vaccines in the US-December 14, 2020-January 18, 2021. JAMA 2021;325:1101-2.

9. World Health Organization (WHO). Statement for healthcare professionals: how COVID-19 vaccines are regulated for safety and effectiveness [Internet]. Geneva: WHO; 2021 Jun 11 [cited 2021 Nov 15]. Available from: https://www.who.int/news/item/11-06-2021statement-for-healthcare-professionals-how-covid-19-vaccinesare-regulated-for-safety-and-effectiveness.

10. World Health Organization (WHO). Coronavirus disease (COVID-19): vaccine [Internet]. Geneva: WHO [cited 2021 Nov 15]. Available from: https://www.who.int/emergencies/diseases/novel-coronavirus-2019/ covid-19-vaccines. 